\title{
Heart failure risk prediction in the Multi-Ethnic Study of Atherosclerosis
}

\author{
Harjit Chahal, ${ }^{1}$ David A Bluemke, ${ }^{2}$ Colin $0 \mathrm{Wu}_{1}{ }^{3}$ Robyn McClelland, ${ }^{4}$ Kiang Liu, ${ }^{5}$ \\ Steven J Shea, ${ }^{6}$ Gregory Burke, ${ }^{7}$ Pelbreton Balfour, ${ }^{8}$ David Herrington, ${ }^{8}$ PeiBei Shi, ${ }^{3}$ \\ Wendy Post, ${ }^{1}$ Jean Olson, ${ }^{9}$ Karol E Watson, ${ }^{10}$ Aaron R Folsom, ${ }^{11}$ Joao A C Lima ${ }^{1}$
}

\begin{abstract}
- Additional material is published online only. To view please visit the journal online (http://dx.doi.org/10.1136/ heartjnl-2014-305697).

For numbered affiliations see end of article.
\end{abstract}

\section{Correspondence to}

Dr Joao A C Lima, Department of Cardiology, Johns Hopkins Hospital, 600 North Wolfe Street, Blalock 524D1, Baltimore, MD 21287, USA; jlima@jhmi.edu

Received 22 February 2014 Revised 31 August 2014 Accepted 15 September 2014 Published Online First 7 November 2014

\section{SLinked}

- http://dx.doi.org/10.1136/ heartjnl-2014-306459

\section{CrossMark}

To cite: Chahal $\mathrm{H}_{\text {, }}$ Bluemke DA, Wu CO, et al. Heart 2015;101:58-64.

\section{ABSTRACT}

Objective Heart failure (HF) is a leading cause of mortality especially in older populations. Early detection of high-risk individuals is imperative for primary prevention. The purpose of this study was to develop a HF risk model from a population without clinical cardiac disease.

Methods The Multi-Ethnic Study of Atherosclerosis is a multicentre observational cohort study following 6814 subjects (mean age $62 \pm 10$ years; $47 \%$ men) who were free of clinical cardiovascular disease at baseline. Median follow-up was 4.7 years. HF events developed in 176 participants. Cox proportional hazards models and regression coefficients were used to determine independent risk factors and generate a 5 -year risk score for incident HF. Bootstrapping with bias correction was used for internal validation.

Results Independent predictors for HF (HR, p value) were age (1.30 (1.10 to 1.50) per 10 years), male gender (2.27 (1.53 to 3.36)), current smoking (1.97 (1.15 to 3.36$))$, body mass index (1.40 (1.10 to 1.80$)$ per $\left.5 \mathrm{~kg} / \mathrm{m}^{2}\right)$, systolic blood pressure (1.10 (1.00 to 1.10) per $10 \mathrm{~mm} \mathrm{Hg}$ ), heart rate (1.30) (1.10 to 1.40$)$ per $10 \mathrm{bpm})$, diabetes (2.27 (1.48 to 3.47)), N-terminal pro-B-type natriuretic peptide (NT proBNP) (2.48 (2.16 to 2.84 ) per unit log increment) and left ventricular mass index (1.40 (1.30 to 1.40$)$ per $\left.10 \mathrm{~g} / \mathrm{m}^{2}\right)$. A parsimonious model based on age, gender, body mass index, smoking status, systolic blood pressure, heart rate, diabetes and NT proBNP natriuretic peptide predicted incident HF risk with a c-statistic of 0.87 .

Conclusions A clinical algorithm based on risk factors readily available in the primary care setting can used to identify individuals with high likelihood of developing HF without pre-existing cardiac disease.

\section{INTRODUCTION}

Heart failure (HF) is a leading cause of morbidity and mortality in the USA and worldwide despite clinical advances made in the diagnosis and treatment of cardiovascular disease. ${ }^{1}$ It is a progressive multiorgan system disease associated with very high admission and readmission rates and ultimately shortened life expectancy in older adults. ${ }^{2-4}$ Worldwide, the incidence and prevalence of HF continues to rise due to improved life expectancy and survival from acute coronary diseases. ${ }^{5}$ Given the immense burden and healthcare costs associated with HF, the American College of Cardiology/
American Heart Association (ACC/AHA) guidelines reclassified HF to include stage A which includes patients with risk factors but no structural heart disease. $^{6}$

The Multi-Ethnic Study of Atherosclerosis (MESA) is a multicentre prospective cohort study designed to investigate the prevalence, correlates and progression of subclinical cardiovascular disease in community dwelling adults without previous clinical cardiovascular disease. ${ }^{7}$ In this study, we sought to determine a parsimonious risk prediction equation for use in primary care setting to identify individuals at high risk for developing HF (see online supplement).

\section{METHODS}

\section{Study design}

The study design, sampling method and data collection have been described in detail elsewhere. ${ }^{7}$ The participants of MESA were 6814 men and women aged 45-84 years old who were Caucasian, African-American, Hispanic or Chinese-American. Exclusion criteria included clinical cardiovascular disease, current atrial fibrillation, any cardiovascular procedure, pregnancy, active cancer treatment, weight $>136 \mathrm{~kg}$ and serious medical condition that precluded long-term participation (see online supplement). The protocols of MESA were approved by the Institutional Review Boards of all collaborating institutions and the National Heart, Lung and Blood Institute and all participants signed informed consent.

\section{Covariates}

Race/ethnicity was defined by self-report during the baseline MESA exam according to 2000 US Census. Standard questionnaires were used to ascertain smoking (classified as never, former and current). Body mass index (BMI) was calculated by dividing weight in kilograms by the square of height in metres. Resting blood pressure was measured using the Dinamap Monitor PRO 100 (Critikon, Tampa, Florida, USA) automated oscillometric device. Serum glucose, total and highdensity lipoprotein cholesterol were measured from blood samples after 12-h fast. The diagnosis of diabetes mellitus was based on the use of insulin or oral hypoglycaemic medication or fasting glucose $\geq 126 \mathrm{mg} / \mathrm{dL}$. Impaired fasting glucose was considered present if fasting glucose was between $100-125 \mathrm{mg} / \mathrm{dL}$. Low-density lipoprotein cholesterol 
was calculated with the Friedewald equation. ${ }^{8}$ Serum creatinine was measured using colorimetry with a Johnson \& Johnson Vitros 950 analyser (Johnson \& Johnson Clinical Diagnostics, Rochester, New York, USA) and calibrated to the Cleveland Clinic standard. Left ventricle (LV) hypertrophy by electrocardiogram was defined using standard criteria. ${ }^{9}$ A subcohort of 5597 participants underwent plasma $\mathrm{N}$-terminal pro-B-type natriuretic peptide (NT-proBNP) measurements. ${ }^{10} \mathrm{LV}$ mass was determined by cardiac magnetic resonance imaging and indexed to body surface area. ${ }^{11}$ Myocardial infarction was diagnosed based on a combination of symptoms, electrocardiogram and cardiac biomarker levels. Interim myocardial infraction was defined as "newly diagnosed acute myocardial infarction during the follow-up period". ${ }^{12}$

\section{HF events}

Participants were re-examined approximately every other year after the baseline examination. ${ }^{13}$ In addition to the study examinations, a telephone interviewer contacted each participant every 9-12 months to inquire about all interim hospital admissions, cardiovascular outpatient diagnoses and deaths. To verify self-reported diagnoses, copies were requested of all death certificates and medical records for all hospitalisations and outpatient cardiovascular diagnoses. Next-of-kin interviews for out-of-hospital cardiovascular deaths were obtained. We were successful in getting medical records on an estimated $98 \%$ of hospitalised cardiovascular events and information on $95 \%$ of outpatient cardiovascular diagnostic encounters. Follow-up telephone interviews were completed by $92 \%$ of living participants. ${ }^{12}$ Trained personnel abstracted any hospital records suggesting possible cardiovascular events and transmitted these to the coordinating centre. These were then sent to two paired physicians (cardiologists or cardiovascular physician epidemiologists) for independent end point classification and assignment of incidence dates. Persisting disagreements were classified by the full review committee. The end point for this study was a composite of probable and definite HF. Definite or probable HF required HF symptoms, such as shortness of breath or oedema. In addition to symptoms, classification of probable HF required diagnosis of HF by a physician and a receipt for medical treatment for HF. Definite HF also required one or more objective criteria, such as pulmonary oedema/congestion by chest X-ray; dilated ventricle or poor LV function by echocardiography or ventriculography; or evidence of $\mathrm{LV}$ diastolic dysfunction. ${ }^{14}$ For this analysis, we used incident definite or probable $\mathrm{HF}$ as a single entity without subdividing it into systolic or diastolic predominance.

\section{Statistical analysis}

For evaluating the covariate effects on time to HF during the follow-up, we treated death or loss to follow-up as censored, and considered five different Cox proportional hazards ( $\mathrm{PH})$ models, each with a different set of risk factors. The simplest model, Model 1, includes only the basic risk factors; age, gender, ethnicity, BMI, among others, while the subsequent models included additional variables. We evaluated the inclusion of significant covariates in the Cox PH models using least absolute shrinkage and selection operator variable selection technique. ${ }^{15}$ Natural log-transformations were applied to variables with skewed distributions. Hazard ratios for the development of HF and their 95\% confidence intervals were computed for each of the risk factors based on their corresponding Cox $\mathrm{PH}$ models. In addition to the Cox PH models, we also examined the potential non-linear effects of several continuous covariates on the incidence of HF using local polynomial smoothing fits. For the variables entering the final Cox PH model, we evaluated their interactions with age, gender and ethnicity after correction for multiple testing. Internal validation of the risk prediction model was done using the bootstrap method with bias correction employing the ' 0.632 bootstrap'. ${ }^{16}$ Bootstrapping is a standard method for evaluating the prediction errors of risk prediction models by comparing the prediction errors from the original sample and the bootstrap samples, which are drawn with replacement from the original sample. Since the outcome of a subject being predicted may also appear in a bootstrap sample used to construct the prediction model, the ' 0.632 bootstrap' is a modified bootstrap method which adjusts the potential bias by using only the bootstrap samples which do not contain the subject being predicted. As shown by various simulation and numerical studies, the ' 0.632 bootstrap' method has the best performance in the estimation of model prediction errors among cross-validation and different bootstrap methods. The Harrell-Pencina-D'Agostino survival c-statistics were used to compare the adequacy of different HF risk prediction models. ${ }^{17}$ Goodness of fit of the statistical models was assessed by the Hosmer-Lemeshow $\chi^{2}$ statistic. Risk reclassification for the HF risk prediction models was evaluated and compared using the net reclassification improvement (NRI) methods. ${ }^{18}$ For the computations of NRI, we considered the actual HF events and predicted HF probabilities based on the Cox PH models. The HF risk classification was defined to be low, intermediate, high and very high if the probability of developing $\mathrm{HF}$ was $<5 \%$, $5-10 \%, 10-20 \%$ and $>20 \%$, respectively, similar to risk categories used in other studies. ${ }^{19-21}$ For easy use to clinicians and patients, the results from the above models were used to create a point-based scoring system similar to the Framingham Heart Study model to generate risk estimates of HF development over a 5 -year period. ${ }^{22}$ In brief, risk factors were organised into meaningful categories and a referential group identified. The difference between each category and the reference category in terms of regression units was computed. A constant corresponding to each unit point increase was defined. The points associated with each risk category were determined from the risk category, its reference value and the constant. The total points and associated risk were thus calculated.

\section{RESULTS}

\section{Study population}

The mean age of MESA participants at baseline was $62 \pm 10$ years with $47.2 \%$ male participants; $38.5 \%$ Caucasian, $11.8 \%$ Chinese, 27.8\% African-American and 22\% Hispanic. Forty-five percent $(45 \%)$ of MESA participants had hypertension, $12.6 \%$ had diabetes mellitus, $13.8 \%$ had impaired fasting glucose, $13 \%$ were current smokers, $32.2 \%$ were obese (BMI $>30 \mathrm{~kg} / \mathrm{m}^{2}$ ) and $39.2 \%$ were overweight (BMI $25-29.9 \mathrm{~kg} / \mathrm{m}^{2}$ ). At baseline, $16 \%$ were on lipid lowering medication and $37 \%$ on antihypertensive therapy.

\section{HF events}

Following the baseline data collected from 2000 to 2002, we used the incident $\mathrm{HF}$ events that occurred subsequently during the 7.1 years of follow up. Clinical HF developed in 176 MESA participants in 4.7 years of median follow-up (range 0.1-7.0 years). Baseline demographic and risk factor characteristics of 
Table 1 Baseline characteristics of participants who developed HF versus those who did not develop HF at 7.1 years follow-up in MESA

\begin{tabular}{|c|c|c|c|}
\hline & $\begin{array}{l}\text { Overall } \\
(n=6814)\end{array}$ & Developed HF at follow-up $(n=176)$ & No HF at follow-up ( $n=6633$ ) \\
\hline Age (years) & $62.1 \pm 10$ & $69.1 \pm 10^{*}$ & $62.0 \pm 10$ \\
\hline Male (\%) & 47.1 & $60.8^{*}$ & 46.8 \\
\hline Caucasians (\%) & $2622(38.5)$ & $69(39.2)$ & $2553(38.4)$ \\
\hline Chinese-Americans (\%) & $803(11.7)$ & $9(5.1)^{*} \dagger$ & $794(12.0)$ \\
\hline African-Americans (\%) & $1892(27.8)$ & $59(33.5)^{*} \dagger$ & $1833(27.6)$ \\
\hline Hispanics (\%) & $1496(22.0)$ & $39(22.2)^{*} \dagger$ & $1457(22.0)$ \\
\hline Systolic blood pressure (mm $\mathrm{Hg}$ ) & $126.6 \pm 21.5$ & $140.4 \pm 22.4^{*}$ & $126.2 \pm 21.3$ \\
\hline Diastolic blood pressure $(\mathrm{mm} \mathrm{Hg})$ & $71.9 \pm 10.3$ & $73.9 \pm 10.8^{*}$ & $71.9 \pm 10.2$ \\
\hline Heart rate $(\mathrm{bpm})$ & $63 \pm 10$ & $65.0 \pm 11.1^{*}$ & $63.1 \pm 9.6$ \\
\hline Total cholesterol (mg/dL) & $194 \pm 35.7$ & $189.65 \pm 35.1$ & $194.3 \pm 35.7$ \\
\hline HDL cholesterol (mg/dL) & $51 \pm 15$ & $47.9 \pm 14.1^{*}$ & $51.1 \pm 14.8$ \\
\hline Hypertension (\%) & 44.9 & $75.6^{*}$ & 44.1 \\
\hline Hypertension medication (\%) & 37.2 & $63.1^{*}$ & 36.5 \\
\hline Diabetes mellitus (\%) & 12.6 & $31.8^{*}$ & 12.1 \\
\hline Current cigarette smokers (\%) & 13.0 & $16.5^{*}$ & 12.9 \\
\hline Pack years & $11.4 \pm 22.3$ & $18.3 \pm 30.9^{*}$ & $11.2 \pm 22.0$ \\
\hline BMI $\left(\mathrm{kg} / \mathrm{m}^{2}\right)$ & $28.3 \pm 5.5$ & $30.1^{*}$ & 28.3 \\
\hline Creatinine (mg/dL) & $0.96 \pm 0.3$ & $1.1 \pm 0.7^{*}$ & $0.9 \pm 0.3$ \\
\hline ECG-LVH (\%) & 1.0 & $6.3^{*}$ & 0.9 \\
\hline LV mass index $\left(\mathrm{g} / \mathrm{m}^{2}\right)$ & $77.9 \pm 16.3$ & $96.7^{*}$ & 77.5 \\
\hline NT-proBNP (pg/mL) & $102.3 \pm 249.7$ & $456.4^{*}$ & 93.0 \\
\hline
\end{tabular}

Parentheses represent units of measurements with the respective $95 \% \mathrm{Cls}$ or percentages as appropriate.

${ }^{*} \mathrm{p}<0.05$ for difference

tvs Caucasians.

BMI, body mass index; ECG, electrocardiogram; HDL, high-density lipoprotein; HF, heart failure; LVH, LV hypertrophy; MESA, Multi-Ethnic Study of Atherosclerosis;

NT-proBNP, N-terminal pro-B-type natriuretic peptide.

the study participants stratified by incident $\mathrm{HF}$ are presented in table 1. Participants who developed HF were more likely at baseline to be elderly, male, African-American, hypertensive, diabetic, current smokers and had higher BMI, LV mass index and NT-proBNP.

\section{Risk factors for prediction of incident HF}

Results from the Cox PH models for incident HF are presented in table 2. Significant independent predictors in the basic model (model 1) were age, male gender, smoking status, BMI, systolic blood pressure, heart rate, diabetes mellitus and creatinine.

Table 2 HRs (95\% Cls) associating risk factors with incident HF events in MESA

\begin{tabular}{|c|c|c|c|c|c|c|}
\hline Models (n) & Model 1 (6678) & Model 2 (6678) & Model 3 (5504) & Model 4a (6678) & Model 4b (4912) & Model 5 (4904) \\
\hline Age (per year increment) & $1.07^{1}(1.05$ to 1.09$)$ & $1.08^{1}(1.05$ to 1.10$)$ & $1.03^{2}(1.0$ to 1.05$)$ & $1.07^{1}(1.05$ to 1.09$)$ & $1.08^{1}(1.05$ to 1.10$)$ & $1.04^{1}$ (1.03 to 1.08$)$ \\
\hline Gender (male vs female) & $1.64^{2}(1.15$ to 2.34$)$ & $2.08(1.45$ to 2.99$)$ & $2.27^{1}(1.53$ to 3.36$)$ & $1.55^{2}$ (1.09 to 2.22$)$ & $1.10(0.68$ to 1.77$)$ & 1.36 (0.78 to 2.36$)$ \\
\hline $\begin{array}{l}\text { Cigarette smoking (current } \\
\text { vs never) }\end{array}$ & $2.35^{1}(1.49$ to 3.71$)$ & $2.98^{1}(1.87$ to 4.76$)$ & $1.97^{2}(1.15$ to 3.36$)$ & $2.40^{1}(1.54$ to 3.81$)$ & $1.98^{2}(1.13$ to 3.46$)$ & $2.54^{2}(1.36$ to 4.74$)$ \\
\hline BMI (per $\mathrm{kg} / \mathrm{m}^{2}$ increment) & $1.05^{1}(1.02$ to 1.09$)$ & $1.08^{1}(1.04$ to 1.11$)$ & $1.04^{2}$ (1.0 to 1.08$)$ & $1.05^{2}(1.02$ to 1.08$)$ & - & - \\
\hline Systolic BP (per mm Hg) & $1.02^{1}(1.01$ to 1.02$)$ & $1.01^{1}(1.00$ to 1.02$)$ & $1.0^{3}(1.0$ to 1.01$)$ & $1.02^{1}(1.00$ to 1.02$)$ & $1.00(0.99$ to 1.01$)$ & $1.00(0.99$ to 1.01$)$ \\
\hline Heart rate (per beat/min) & $1.02^{3}(1.0$ to 1.03$)$ & 1.01 (1.0 to 1.03$)$ & $1.03^{2}(1.0$ to 1.04$)$ & $1.02^{3}(1.00$ to 1.03$)$ & $1.03^{2}(1.01$ to 1.05$)$ & $1.04^{1}$ (1.02 to 1.06$)$ \\
\hline Diabetes (vs non-diabetics) & $2.34^{1}(1.62$ to 3.37$)$ & $1.83^{1}(1.25$ to 2.68$)$ & $2.27^{1}(1.48$ to 3.47$)$ & $2.30^{1}(1.60$ to 3.32$)$ & $2.58^{1}(1.60$ to 4.16$)$ & $1.85^{3}(1.05$ to 3.28$)$ \\
\hline Creatine (per mg/dL) & $1.40^{1}(1.16$ to 1.70$)$ & $0.94(0.78$ to 1.48$)$ & - & $1.42^{1}(1.18$ to 1.71$)$ & $1.28^{3}(1.04$ to 1.57$)$ & - \\
\hline $\begin{array}{l}\text { NT-proBNP (per log unit } \\
\text { increment) }\end{array}$ & & - & $2.48^{1}$ (2.16to 2.84) & - & - & $1.62^{1}(1.35$ to 1.95$)$ \\
\hline ECG-LVH (vs no LVH) & - & - & & $4.16^{1}(2.20$ to 7.85$)$ & - & - \\
\hline $\begin{array}{l}\text { LV mass index (per unit } \\
\mathrm{g} / \mathrm{m}^{2} \text { increment) }\end{array}$ & - & - & & - & $1.04^{1}(1.03$ to 1.04$)$ & $1.02^{1}(1.01$ to 1.03$)$ \\
\hline
\end{tabular}

${ }^{1} p<0.001,{ }^{2} p<0.01,{ }^{3} p<0.05$.

Significant HR and their confidence intervals are presented in bold.

Model 1: includes age, gender, ethnicity, BMI, cigarette smoking, systolic blood pressure, heart rate, diabetes mellitus, total cholesterol, HDL cholesterol and creatine.

Model 2: Model 1+ interim myocardial infarction.

Model 3: age, gender, ethnicity, BMI, cigarette smoking, systolic blood pressure, heart rate, diabetes mellitus, total cholesterol, HDL cholesterol and log of NT-proBNP.

Model 4a: age, gender, ethnicity, BMI, cigarette smoking, systolic blood pressure, heart rate, diabetes mellitus, creatine, total cholesterol, HDL cholesterol and ECG-LVH.

Model 4b: age, gender, ethnicity, cigarette smoking, systolic blood pressure, heart rate, diabetes mellitus, creatine and LV mass indexed to body surface area.

Model 5: age, gender, ethnicity, cigarette smoking, systolic blood pressure, heart rate, diabetes mellitus, interim myocardial infarction, LV mass indexed to body surface area and log of NT-proBNP.

BMI, body mass index; HF, heart failure; LVH, LV hypertrophy; MESA, Multi-Ethnic Study of Atherosclerosis; NT-proBNP, N-terminal pro-B-type natriuretic peptide. 
Harrell's c-statistic for the basic model was 0.80. C-statistic represents the ability of the score to discriminate future events from non-events. In this case a c-statistic of 0.80 indicates that a randomly selected $\mathrm{HF}$ event has a higher score than a randomly selected non-event $80 \%$ of the time. Model 2 was adjusted for interim myocardial infarction. In model 3 , we added log transformed NT-proBNP. Given the collinearity between NT-proBNP and creatine, they were not included in the same model. The statistical associations between risk factors from the base model persisted even after the addition of log NT-proBNP with substantial improvement of c-statistic to 0.87 . When LV mass index was added to the model (model $4 \mathrm{~b}$ ), gender and systolic blood pressure became non-significant; c-statistic for this model was 0.85 . When Left ventricle (LV) hypertrophy by electrocardiogram hypertrophy (model 4a) was used instead of LV mass index, the c-statistic was only marginally higher than the basic model (0.81). For model containing all significant risk predictors (model 5) the c-statistic was 0.91 with age, smoking, heart rate, diabetes, interim myocardial infarction, log NT-proBNP and LV mass index retaining independent statistical significance.

The associations between incident HF and systolic blood pressure, heart rate and LV mass index were non-linear (online supplementary figures 1 and 2). Significant interactions were present for gender by heart rate and LV mass index by ethnicity. For a unit increment in heart rate, women had higher risk for HF than men (HR 1.03 vs 1.01; p for the interaction term, $\mathrm{p}=0.004)$. For a unit increment in LV mass index, the risk for $\mathrm{HF}$ in African-Americans was lower than in Caucasians (HR 1.03 vs 1.05 per $\mathrm{g} / \mathrm{m}^{2} ; \mathrm{p}=0.002$ ).

We assessed the reclassification value of $\log$ NT-proBNP and LV mass index beyond the known risk predictors of HF, including demographics and traditional risk factors (table 3). NRI estimates improved with the addition of log NT-proBNP and LV mass index to traditional risk factors primarily due to upward reclassification of individuals who subsequently developed HF (online supplement 2-5). When LV mass index was added to the model containing traditional risk factors and log NT-proBNP, the NRI estimate for the low, intermediate and high categories was non-significant. Conversely, the addition of $\log$ NT-proBNP improved the NRI beyond LV mass index for the three risk categories.

A point based scoring system was used to develop a method for calculating an associated 5-year HF risk based on age, gender, BMI, smoking status, systolic blood pressure, heart rate, diabetes and NT pro-BNP (tables 4 and 5). ${ }^{22}$ This final MESA HF risk score had a c-statistic of 0.87 (95\% CI 0.82 to 0.88$)$, c-statistic of 0.87 and Hosmer-Lemeshow $\chi^{2}=4.84, p=0.86$.

Table 3 Assessment of risk reclassification from magnetic resonance determined LV mass index (LVMI) vs log NT-proBNP using net reclassification method in MESA

\begin{tabular}{lll}
\hline Models & c-Statistic & NRI ( $p$ value) \\
\hline Model 1 & 0.80 & \\
Model 1+LVMI & 0.85 & $0.25(<0.001)$ \\
Model 1+ log NT-proBNP & 0.87 & $0.37(<0.001)$ \\
(Model 1, LVMI)+ log NT-proBNP & 0.89 & $0.15(0.018)^{*}$ \\
(Model 1, log NT proBNP)+LVMI & 0.89 & $0.01(0.911)^{*}$ \\
\hline
\end{tabular}

Model 1: age, ethnicity, gender, BMI, cigarette smoking, systolic blood pressure, heart rate, diabetes mellitus, total cholesterol and $\mathrm{HDL}$ cholesterol.

${ }^{*}$ For $\mathrm{HF}$ risk categories $<5 \%, 5-20 \%$ and $>20 \%$.

HF, heart failure; MESA, Multi-Ethnic Study of Atherosclerosis; NRI, net reclassification improvement; NT-proBNP, N-terminal pro-B-type natriuretic peptide.
Table 4 Point scoring for calculation of risk factor profile

\begin{tabular}{|c|c|c|c|c|c|}
\hline $\begin{array}{l}\text { Risk } \\
\text { Factor }\end{array}$ & Categories & Points & Risk factor & Categories & Points \\
\hline \multirow[t]{4}{*}{$\begin{array}{l}\text { Age } \\
\text { (years) }\end{array}$} & $45-54$ & 0 & $\begin{array}{l}\text { Systolic } \\
\text { blood } \\
\text { pressure }\end{array}$ & $<120$ & 0 \\
\hline & $55-64$ & 2 & & $120-139$ & 1 \\
\hline & $65-74$ & 4 & & $140-159$ & 2 \\
\hline & $75-84$ & 6 & & $\geq 160$ & 2 \\
\hline \multirow[t]{5}{*}{ Gender } & Female & 0 & Heart rate & $<60$ & -1 \\
\hline & Male & 2 & & $60-69$ & 0 \\
\hline & & & & $70-79$ & 1 \\
\hline & & & & $80-89$ & 2 \\
\hline & & & & $\geq 90$ & 4 \\
\hline \multirow[t]{3}{*}{ Smoker } & Never & 0 & Diabetes & normal & 0 \\
\hline & Former & 0 & & IFG & 1 \\
\hline & Current & 2 & & Diabetes & 2 \\
\hline \multirow[t]{5}{*}{ BMI } & Normal & 0 & NT pro-BNP & $<200 \mathrm{pg} / \mathrm{mL}$ & 0 \\
\hline & Overweight & 0 & & $200-<400 \mathrm{pg} / \mathrm{mL}$ & 4 \\
\hline & BMI Obese & 1 & & $400-<600 \mathrm{pg} / \mathrm{mL}$ & 6 \\
\hline & & & & $600-<800 \mathrm{pg} / \mathrm{mL}$ & 8 \\
\hline & & & & $>800 \mathrm{pg} / \mathrm{mL}$ & 11 \\
\hline
\end{tabular}

BMI, body mass index; SBP, systolic blood pressure; IFG, impaired fasting glucose; NT-proBNP, N-terminal pro-B-type natriuretic peptide.

Addition of NT-proBNP improved the net reclassification index by 0.37 . The distribution of expected versus observed HF incidence across deciles of risk is shown in figure 1.

\section{DISCUSSION}

In this prospective study, we concluded that the model containing traditional risk factors, interim myocardial infarction, LV mass index and log NT-proBNP had the highest discriminative ability to predict incident HF. However a more parsimonious approach using clinical parameters readily available in the primary care setting (age, gender, BMI, smoking status, systolic blood pressure, heart rate, diabetes and NT pro-BNP) also provided a robust predictive algorithm. A point based scoring system based on these risk factors was developed to estimate a 5 -year risk of developing HF from a multiethnic sample of men and women without clinical cardiovascular disease at baseline. The overall risk prediction equation had an excellent discrimination index, good overall calibration and significantly improved reclassification by the addition of NT-proBNP.

The selection of risk factors to be included in the final model was based on their access in clinical practice, ability to improve discrimination (c-statistic) and risk reclassification (NRI) so as to come up with a meaningful and relevant risk assessment profile that translates into clinical decision making. In the MESA study, NT-proBNP and LV mass index have been shown to be powerful

Table 5 MESA 5-year HF risk score

\begin{tabular}{lll}
\hline Cumulative points & HF risk group & 5-year HF risk (\%) \\
\hline$\leq 9$ points & Low & $<5$ \\
$10-12$ points & Average & $5-10 \%$ \\
$12-14$ points & High & $10-20 \%$ \\
$\geq 15$ points & Very high & $>20 \%$ \\
\hline
\end{tabular}

HF, heart failure; MESA, Multi-Ethnic Study of Atherosclerosis. 


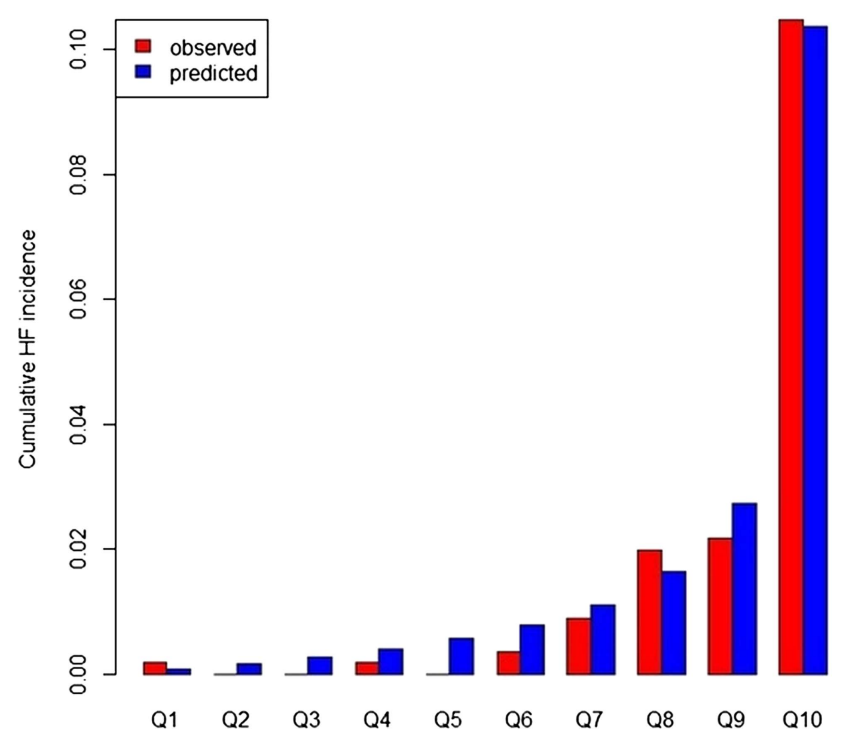

Figure 1 Quintile plots for observed versus predicted risk.

predictors of HF. ${ }^{10}{ }^{12}$ In the present study, the addition of log NT-proBNP produced the largest increase in the c-statistic from the base model. Furthermore, although LV mass was an independent predictor of $\mathrm{HF}$ in all models (including log NT-proBNP); it did not represent a significant risk reclassifier over and above NT-proBNP. On the other hand, NT-proBNP significantly improved the reclassification over LV mass. Therefore we selected NT-proBNP over imaging parameters for the final risk prediction equation.

Risk assessment is the key in the field of cardiovascular disease prevention. In clinical practice, risk prediction algorithms have been used to identify high-risk individuals for more intensive preventive and therapeutic interventions. The MESA HF risk assessment model builds upon the risk evaluation indices derived previously from the Framingham, Health Aging and Body Composition (Health ABC) study and atherosclerosis risk in communities (ARIC) study. ${ }^{19} 2123$ The Framingham risk score is derived from a mostly White population and includes estimates of HF risk in those with coronary heart disease and valvular heart disease using traditional cardiovascular risk factors. While the Framingham equations have been appropriately used widely, they may overestimate risk in ethnicities other than Caucasians and African-Americans. ${ }^{24} 25$ Inherent cardiovascular disease risk in certain ethnic groups and their temporal trends could partly explain these observations. The more recent biethnic ARIC HF risk score also includes prevalent coronary and valvular heart disease in the predictive models. The ARIC HF score showed an improved prediction over the Framingham score by the addition of NT-proBNP. However, it is to be noted that pre-existing coronary and valvular heart diseases in these cohorts characterise them as being at an increased risk for incident HF. The progression of cardiac remodelling and dysfunction that occurs in HF has many diverse pathways (ischaemic, non-ischaemic, valvular). Therefore, the predictive value of a set of risk markers is highly dependent on the population from which it is derived; as well as the population to which it is applied. A score derived from populations that included presence of clinical heart disease at study onset may not be as useful to identify ACC/AHA stage A or stage B HF in populations of healthier individuals. In the Health $\mathrm{ABC}$ study, the risk calculator was derived from a healthier cohort of older adults without pre-existing cardiovascular disease, but that study did not include NT-proBNP, which in MESA and ARIC provided the largest contribution to risk assessment. The MESA study demonstrates a strong predictive model in a multiethnic population without pre-existing clinical cardiac disease that should be used to predict incident HF for primary prevention.

The use of the MESA risk assessment profile based on routine labs could facilitate identification of patients at highest risk levels in whom further testing and aggressive risk reduction could be beneficial. The risk factors included in our study are easily available in primary care settings and some are potentially modifiable. The present study also alludes to certain gender and ethnic differences in relation to risk factors and development of HF. The associations of risk factors with HF were similar across different age, ethnicity and gender groups except heart rate which showed a stronger risk for HF in women as compared with men; and LV mass index which showed a lower risk of HF in African-Americans as compared with Caucasians. Although beyond the scope of this study, such subtle findings could mean differential pathophysiology and/or remodelling in different subgroups in relationship to these risk factors which could be a focus for more specific risk reduction strategies.

Strengths of MESA include high quality assessment of risk markers such as LV mass using magnetic resonance imaging, and the measurement of multiple risk factors including biomarkers which are responsible for a high predictive value of the MESA score. MESA HF events included inpatient and outpatient diagnoses further reviewed and verified by a MESA cardiologist. The MESA population includes a substantial proportion of previously understudied ethnic groups and is derived from diverse geographical locations in the USA.

\section{Key messages}

\section{What is already known on this subject?}

Heart failure (HF) continues to be the leading cause of morbidity and mortality with enormous economic burden globally. Several epidemiological studies have identified cardiac (hypertension, diabetes, obesity, smoking, etc.) and non-cardiac (albumin, renal function, $\mathrm{C}$ reactive protein, etc.) risk factors associated which are associated with the development of HF. Major cohort studies including the older Framingham study and the more recent Atherosclerosis Risk in Communities and the Health $A B C$ studies have devised risk prediction algorithms based on several risk markers to predict future risk of $\mathrm{HF}$.

\section{What might this study add?}

In this study which is derived from the Multi-Ethnic Study of Atherosclerosis (MESA) we developed a point based scoring system for estimating the risk of developing HF based on common risk factors which can be easily available in the primary care setting. We believe that this 'MESA HF-risk score' will be helpful to primary care clinicians and cardiologists to identify and focus on patients that do not have cardiac disease at present, but are at high risk of developing HF in the future.

How might this impact on clinical practice?

Given the high burden, mortality and morbidity associated with $\mathrm{HF}$, experts have recommended greater focus on prevention. Application of a risk prediction tool by clinicians can help motivate patients and physicians to aggressively target modifiable risk factors. 
There are several limitations to our study that deserve discussion. The number of HF events was lower compared with other cohorts primarily due to the younger age of this population. Chinese-American participants had the lowest number of events, making it difficult to derive meaningful associations in this ethnic group. HF was not differentiated into systolic and diastolic HF due to the limited power for subanalysis given the low number of events. Risk factors were measured only at baseline, hence temporal and causal inferences cannot be made from this study alone. Although the ethnic diversity is a unique strength of our study, the MESA population does not represent all ethnic groups. Furthermore, it must be noted that although one of the strengths of MESA is its ethnic diversity and that ethnic differences in HF are well known; ethnicity was not significant in the final model and though available readily at the bedside does not contribute to predicting HF risk in a community population. Lastly, residual confounding cannot be completely eliminated from epidemiological studies.

In conclusion, we derived a robust HF risk estimation tool using clinical and ancillary variables that are partly modifiable and could be targeted for preventive strategies. Future clinical trials designed to examine the effectiveness of HF risk reduction strategies based on this predictive model are warranted.

\section{Author affiliations}

'Department of Cardiology, Johns Hopkins University, Baltimore, Maryland, USA ${ }^{2}$ Department of Radiology and Imaging Sciences, National Institutes of Health, Bethesda, Maryland, USA

${ }^{3}$ Offices of Biostatistics Research, National Heart Lung and Blood Institute, Bethesda, Maryland, USA

${ }^{4}$ Collaborative Health Studies Coordinating Center, University of Washington, Seattle, Washington, USA

${ }^{5}$ Department of Preventive Medicine, Northwestern University Medical School, Chicago, Illinois, USA

${ }^{6}$ Department of Epidemiology, Columbia University, New York, New York, USA

${ }^{7}$ Department of Public Health Sciences, Wake Forest University, Winston-Salem, North Carolina, USA

${ }^{8}$ Department of Cardiology, Wake Forest University, Winston-Salem, North Carolina, USA

${ }^{9}$ Division of Prevention and Population Sciences, National Heart, Lung, and Blood Institute, Bethesda, Maryland, USA

${ }^{10}$ Division of Cardiology, UCLA-School of Medicine, Los Angeles, California, USA

${ }^{11}$ Division of Epidemiology and Community Health, University of Minnesota,

Minneapolis, Minnesota, USA

Funding The Multi-Ethnic Study of Atherosclerosis is sponsored by contracts from the National Heart Lung and Blood Institute (N01-HC-95159 through N01-HC-95166 and N01-HC95169).

Contributors $\mathrm{JACL}$ is the guarantor of the entire manuscript. HC: contributed to study concept and design, analysis and interpretation of data, drafting of the manuscript, and critical revision of the manuscript for important intellectual content, and takes responsibility for the integrity of the work as a whole. DAB: contributed to acquisition and interpretation of MRI data, study concept and design, analysis and interpretation of data, and critical revision of the manuscript for important intellectual content. COW: contributed to study concept and design, analysis and interpretation of data, and critical revision of the manuscript for important intellectual content. RM: contributed to study concept and design, analysis and interpretation of data, and critical revision of the manuscript for important intellectual content. KL: contributed to study concept and design, analysis and interpretation of data, and critical revision of the manuscript for important intellectual content. SJS: contributed to study concept and design, analysis and interpretation of data, and critical revision of the manuscript for important intellectual content. GB: contributed to study concept and design, analysis and interpretation of data, and critical revision of the manuscript for important intellectual content. PB: contributed to acquisition and interpretation of MRI data study concept and design, and critical revision of the manuscript for important intellectual content. DH: contributed to acquisition and interpretation of MRI data study concept and design, and critical revision of the manuscript for important intellectual content. PS: contributed to statistical analysis and interpretation of data, drafting of the manuscript, and critical revision of the manuscript for important intellectual content. WP: contributed to study concept and design, analysis and interpretation of data, drafting of the manuscript, and critical revision of the manuscript for important intellectual content. J0: contributed to study concept and design, analysis and interpretation of data, drafting of the manuscript, and critical revision of the manuscript for important intellectual content. KEW: contributed to study concept and design, analysis and interpretation of data, drafting of the manuscript, and critical revision of the manuscript for important intellectual content. ARF: contributed to study concept and design, analysis and interpretation of data, drafting of the manuscript, and critical revision of the manuscript for important intellectual content. JACL: contributed to acquisition and interpretation of MRI data, study concept and design, analysis and interpretation of data, and critical revision of the manuscript for important intellectual content.

Competing interests $H C, D A B, C O W, R M, K L, S J S, G B, P B, D H, P S, W P, J O$, KEW, ARF and JACL have reported that no potential conflicts of interest exist with any companies/organisations whose products or services may be discussed in this article. This manuscript was reviewed by MESA investigators for scientific content and consistency of data interpretation with previous MESA publications, and significant comments were incorporated before submission for publication. The authors thank the other investigators, staff, and participants of the MESA studies for their valuable contributions. A full list of participating MESA investigators and institutions can be found at http://www.mesa-nhlbi.org.

Ethics approval Institutional Review Boards of all collaborating institutions and the National Heart, Lung and Blood Institute (NHLBI).

Provenance and peer review Not commissioned; externally peer reviewed.

Data sharing statement The corresponding author has the right to grant on behalf of all authors and does grant on behalf of all authors, an exclusive license (or non-exclusive for government employees) on a worldwide basis to the BMJ Publishing Group and its licensees to permit this article (if accepted) to be published in HEART editions and any other BMJPGL products to exploit all subsidiary rights.

\section{REFERENCES}

1 Lloyd-Jones D, Adams RJ, Brown TM, et al. Heart disease and stroke statistics - 2010 update: a report from the American Heart Association. Circulation 2010;121:e46-215

2 Krumholz HM, Chen YT, Wang Y, et al. Predictors of readmission among elderly survivors of admission with heart failure. Am Heart J 2000;139:72-7.

3 Wexler DJ, Chen J, Smith GL, et al. Predictors of costs of caring for elderly patients discharged with heart failure. Am Heart J 2001;142:350-7.

4 Neubauer $S$. The failing heart-an engine out of fuel. $N$ Engl J Med 2007;356:1140-51.

5 Roger VL, Weston SA, Redfield MM, et al. Trends in heart failure incidence and survival in a community-based population. JAMA 2004;292:344-50.

6 Hunt SA, Baker DW, Chin MH, et al. ACC/AHA Guidelines for the Evaluation and Management of Chronic Heart Failure in the Adult: Executive Summary A Report of the American College of Cardiology/American Heart Association Task Force on Practice Guidelines (Committee to Revise the 1995 Guidelines for the Evaluation and Management of Heart Failure): Developed in Collaboration With the International Society for Heart and Lung Transplantation; Endorsed by the Heart Failure Society of America. Circulation 2001;104:2996-3007.

7 Bild DE, Bluemke DA, Burke GL, et al. Multi-ethnic study of atherosclerosis: objectives and design. Am J Epidemiol 2002;156:871-81.

8 Friedewald WT, Levy RI, Fredrickson DS. Estimation of the concentration of low-density lipoprotein cholesterol in plasma, without use of the preparative ultracentrifuge. Clin Chem 1972;18:499-502.

9 Jain A, Tandri H, Dalal D, et al. Diagnostic and prognostic utility of electrocardiography for left ventricular hypertrophy defined by magnetic resonance imaging in relationship to ethnicity: the Multi-Ethnic Study of Atherosclerosis (MESA). Am Heart J 2010;159:652-8.

10 Choi EY, Bahrami $\mathrm{H}$, Wu CO, et al. N-terminal pro-B-type natriuretic peptide, left ventricular mass, and incident heart failure: Multi-Ethnic Study of Atherosclerosis. Circ Heart Fail 2012;5:727-34.

11 Natori S, Lai S, Finn JP, et al. Cardiovascular function in multi-ethnic study of atherosclerosis: normal values by age, sex, and ethnicity. AJR Am J Roentgenol 2006; 186:S357-65

12 Bluemke DA, Kronmal RA, Lima JA, et al. The relationship of left ventricular mass and geometry to incident cardiovascular events: the MESA (Multi-Ethnic Study of Atherosclerosis) study. J Am Coll Cardiol 2008;52:2148-55.

13 Bahrami $H$, Kronmal R, Bluemke DA, et al. Differences in the incidence of congestive heart failure by ethnicity: the multi-ethnic study of atherosclerosis. Arch Intern Med 2008;168:2138-45.

14 Opdahl A, Ambale Venkatesh B, Fernandes VR, et al. Resting heart rate as predictor for left ventricular dysfunction and heart failure: MESA (Multi-Ethnic Study of Atherosclerosis). J Am Coll Cardiol 2014:63:1182-9.

15 Tibshirani R. The lasso method for variable selection in the Cox model. Stat Med 1997;16:385-95. 
16 Steyerberg EW, Bleeker SE, Moll HA, et al. Internal and external validation of predictive models: a simulation study of bias and precision in small samples. J Clin Epidemiol 2003;56:441-7.

17 Pencina MJ, D'Agostino RB Sr, Song L. Quantifying discrimination of Framingham risk functions with different survival C statistics. Stat Med 2012;31:1543-53.

18 Pencina MJ, D'Agostino RB Sr, D'Agostino RB Jr, et al. Evaluating the added predictive ability of a new marker: from area under the ROC curve to reclassification and beyond. Stat Med 2008:27:157-72; discussion 207-12

19 Butler J, Kalogeropoulos A, Georgiopoulou V, et al. Incident heart failure prediction in the elderly: the health $A B C$ heart failure score. Circ Heart Fail 2008;1:125-33.

20 Smith JG, Newton-Cheh C, Almgren P, et al. Assessment of conventional cardiovascular risk factors and multiple biomarkers for the prediction of incident heart failure and atrial fibrillation. J Am Coll Cardiol 2010;56:1712-9.
21 Agarwal SK, Chambless LE, Ballantyne CM, et al. Prediction of incident heart failure in general practice: the Atherosclerosis Risk in Communities (ARIC) Study. Circ Heart Fail 2012;5:422-9.

22 Sullivan LM, Massaro JM, D'Agostino RB Sr. Presentation of multivariate data for clinical use: The Framingham Study risk score functions. Stat Med 2004;23:1631-60.

23 Kannel WB, D'Agostino RB, Silbershatz $\mathrm{H}$, et al. Profile for estimating risk of heart failure. Arch Intern Med 1999;159:1197-204.

24 D'Agostino RB Sr, Grundy S, Sullivan LM, et al. Validation of the Framingham coronary heart disease prediction scores: results of a multiple ethnic groups investigation. JAMA 2001;286:180-7.

25 Liu J, Hong Y, D'Agostino RB Sr, et al. Predictive value for the Chinese population of the Framingham CHD risk assessment tool compared with the Chinese Multi-Provincial Cohort Study. JAMA 2004;291:2591-9. 